\title{
Diagnosis and treatment of nosocomial pneumonia in ALI/ARDS patients
}

\author{
J-Y. Fagon, J. Chastre
}

Diagnosis and treatment of nosocomial pneumonia in ALI/ARDS patients. J-Y. Fagon, J. Chastre. C) ERS Journals Ltd 2003.

ABSTRACT: Ventilator-associated pneumonia (VAP) is a common complication of the acute respiratory distress syndrome (ARDS) or acute lung injury (ALI), often leading to the development of sepsis, multiple organ failure, and death. However, the diagnosis of pulmonary infection in patients with ARDS/ALI is often difficult: the systemic signs of infection, such as fever, tachycardia, leukocytosis are nonspecific findings in such patients; a variety of causes other than pneumonia can explain asymmetric consolidation in patients with ARDS and marked asymmetry of radiographic abnormalities has also been reported in patients with uncomplicated ARDS.

In 2003, physicians in charge of these patients have to identify patients with true bacterial lung infection, to select appropriate initial antibiotic therapy, to adjust therapy as soon as possible, and to withhold antibiotics in patients without VAP. To do that, a bacteriological strategy based on the use of quantitative cultures of specimen obtained with fibreoptic bronchoscopy performed before initiation or modification of antibiotic treatment seems better than a strategy based on clinical evaluation alone, lowering antibiotic consumption and improving outcome. When bronchoscopy is not available or contraindicated, a nonbronchoscopic strategy or a clinical strategy with reevaluation 3 days after initiation of treatment may be used.

Antimicrobial treatment of VAP is a complex issue. Some general principles can be helpful for the selection of initial treatment: knowledge of most frequently identified responsible pathogens and their susceptibility patterns in the unit; prior duration of hospitalisation; previously prescribed antibiotics; information obtained by direct examination of pulmonary secretions; antibacterial activity and pharmacodynamic characteristics of antibiotics that could be used to treat this infection.

Appropriateness of initial antimicrobial therapy is probably a major prognostic factor for patients with ventilator-associated pneumonia. Thus, before new antiboitics are administered, reliable pulmonary specimens must be obtained for direct examination and cultures.

Eur Respir J 2003; 22: Suppl. 42, 77s-83s.
Services de Réanimation Médicale, Hôpital Européen Georges-Pompidou and Groupe Hospitalier Pitié-Salpêtrière, Paris, France.

Correspondence: J-Y. Fagon

Service de Réanimation Médicale

Hôpital Européen Georges-Pompidou

20 , rue Leblanc

75908 Paris Cedex 15

France

Fax: 33156093202

E-mail: jean-yves.fagon@hop.egp.ap-hop-paris.fr

Keywords: Antibiotic therapy

fibreoptic bronchoscopy

resistant pathogens

ventilator-associated pneumonia
Because ventilator-associated pneumonia (VAP) is a frequent and severe complication occurring in patients treated with mechanical ventilation, rapid identification of infected patients and accurate selection of antimicrobial agents represent important goals [1]. In patients on mechanical ventilation, pneumonia results from microbial invasion of the normally sterile lower respiratory tract. The majority of these infections appear to result from aspiration of potential pathogens that have colonised the oropharyngeal airways. Intubation facilitates the entry of bacteria into the lung by pooling and leakage of contaminated secretions around the endotracheal tube cuff $[2,3]$. Thus, the tracheobronchial tree as well as the oropharynx of mechanically ventilated patients are frequently colonised by enteric Gram-negative bacilli [4-6]. However, the relationship between tracheal colonisation and lung infection remains unclear: JOHANSON et al. demonstrated that only $23 \%$ of colonised patients subsequently developed nosocomial pneumonia [4]. As a consequence, the first major obstacle to be confronted for the diagnosis of VAP is that, unlike patients developing community-acquired pneumonia, the presence of bacteria in the lower airways of intubated patients is not a sufficient argument to diagnose true lung infection.
The diagnosis of pneumonia is usually based on the presence of fever, leukocytosis and new or worsening lung infiltrates on the chest radiograph [7]. These clinical and radiological signs are reliable diagnostic criteria to determine whether community-acquired pneumonia is present in a patient admitted to the emergency room or the intensive care unit (ICU). In contrast, systemic signs of infection and/or lung infiltrates are frequently due to other causes in hospitalised, ventilator-dependent patients. Fever and leukocytosis may be the consequences of any condition that releases cytokines, including noninfectious origins, such as drug-related fever, pulmonary oedema or infarction, and nonpulmonary infections such as vascular catheter infection, gastrointestinal infection, urinary tract infection, postoperative fever, sinusitis or wound infection. MEDURI et al. [8] confirmed the presence of lung infection in only $42 \%$ of the patients with clinically suspected VAP, and the frequent occurrence of multiple infections or noninfectious processes. Similarly, when infiltrates are present on the chest radiograph, it is difficult to differentiate among cardiogenic pulmonary oedema, atelectasis, pulmonary contusion, infarction and pneumonia in a patient treated with mechanical ventilation, frequently admitted to the ICU because of the presence of 
severe pulmonary disease [9]. Finally, purulent secretions are almost inevitably found in patients receiving prolonged mechanical ventilation and do not specifically indicate the presence of pneumonia $[4,10]$.

The second major difficulty for diagnosing VAP is that the presence of signs suggestive of pneumonia in non-ICU patients are too nonspecific to be of diagnostic value for ventilated patients.

The third major problem with the management of patients suspected of having VAP concerns the use of antibiotics. Most epidemiological investigations have clearly demonstrated that the indiscriminate administration of antimicrobial agents to patients in the ICU has immediate and long-term consequences, which contribute to the emergence of multiresistant pathogens, increase the risk of severe super infections with potentially increased morbidity and mortality, in addition to antibiotic-related toxicity and higher costs [11-13].

In 2003, it is essential to treat VAP patients without delay, and to limit the development and dissemination of antibioticresistant infections. Intensivists responsible for patients on mechanical ventilation have: 1) to identify patients with VAP, 2) to select appropriate initial antimicrobial therapy, 3) to adjust therapy as soon as possible, and 4) to withhold antibiotics from patients without VAP. However, to do so, no consensus has yet been reached on the best diagnostic tool for VAP. The major diagnostic controversies revolve around the microbiological diagnosis, principally the need for invasive techniques to obtain specimens and the need for quantitative cultures of these samples. In fact, the question is not the choice between an invasive and a noninvasive diagnostic technique, but between a clinical and a bacteriological strategy.

\section{Evaluation of diagnostic strategies}

The most common strategies applied to diagnose VAP are: 1) clinical strategy alone, 2) clinical strategy with shortcourse antibiotic therapy, 3) clinical strategy using qualitative cultures of tracheal secretions, 4) clinical strategy with quantitative cultures of tracheal secretions, 5) clinical or bacteriological strategy using nonbronchoscopic (blind) techniques, 6) bacteriological strategy using fibreoptic bronchoscopy to obtain protected specimen brush (PSB) and/or bronchoalveolar lavage (BAL) samples.

Numerous high-quality studies have assessed different diagnostic techniques in the setting of suspected VAP, with the determination of their operative indices compared to human or animal histopathological and/or bacteriological definition(s) of pneumonia [14, 15]. In contrast, only a few studies have correctly evaluated strategies, including diagnostic and therapeutic approaches, trying to answer the two major questions: does application of the strategy result in a benefit for the patient? and does application of the strategy result in reducing and controlling overuse of antimicrobial agents?

Before considering the benefits in terms of outcome, clear distinction between strategies and techniques is required because it is possible to apply different strategies with the same diagnostic technique and to apply the same strategy with different diagnostic techniques. The best way to distinguish one strategy from another is to identify differences in the decision to treat, the choice of antimicrobial agents, the decision to withhold treatment, i.e. differences in the prescription of antibiotics. Thus, the most common strategies have to be evaluated with antibiotic consumption and outcome as major endpoints.

\section{Clinical strategy alone}

This approach results in the administration of antibiotics to all patients clinically suspected of having pneumonia. Although the sensitivity of a clinical diagnosis of VAP is high, its specificity is low [16]. As a consequence, the application of clinical criteria alone is unlikely to result in withholding antibiotic therapy from patients who truly have pneumonia. In addition, no bacteriological information is available to aid in the choice of antimicrobial agent(s) and many patients are inappropriately given broad-spectrum antibiotics. The choice of antibiotics is particularly difficult in critically ill patients because VAP is likely to result from highly resistant organisms, such as Pseudomonas aeruginosa, Acinetobacter baumannii, methicillin-resistant Staphylococcus aureus, and/or extended spectrum $\beta$-lactamase-producing Gram-negative bacilli, especially in patients who had previously been treated with antibiotics $[17,18]$, and is frequently (probably in at least $40 \%$ of cases) due to multiple organisms [19, 20]. Therefore, no "magic bullet" exists to cover all the microorganisms potentially responsible for VAP. The use of guidelines from consensus conferences or experts' statements for the choice of initial treatment results in excessive and, frequently, inappropriate or at least nonoptimal usage of antimicrobial therapy [17]. Such a strategy may be improved by tailoring the selection of initial antimicrobial therapy to each institution's local patterns of antimicrobial resistance [20, 21].

With the objective to improve the accuracy of clinical diagnosis, PUGIN et al. [22] developed a clinical pulmonary infection score (CPIS), obtained by according 0 to 2 points for each of 7 variables including semiquantitative cultures of tracheal aspirate. Using BAL as the standard, a CPIS $>6$ had a sensitivity of $93 \%$ and a specificity of $100 \%$ for the diagnosis of VAP, probably lowering the number of unnecessarily prescribed antibiotics [22]. This score remains to be validated in a large prospective study.

More generally, the effectiveness of a strategy based only on clinical evaluation of ventilated patients with fever, leukocytosis, purulent tracheal secretions and lung infiltrates has never been evaluated.

\section{Clinical strategy with a short-course antibiotic therapy}

Recently, SINGH et al. [23] described a strategy in which decisions regarding antibiotic therapy were based on a modified version of the CPIS. Patients with CPIS $>6$ were treated as if they had pneumonia with antibiotics for 10-21 days (standard therapy), patients with CPIS $\leqslant 6$ were randomised to receive either standard therapy or ciprofloxacin monotherapy with re-evaluation 3 days later. Ciprofloxacin was discontinued if CPIS remained $\leqslant 6$ on day 3 ; patients with CPIS $>6$, were treated for pneumonia. Antibiotics were continued for $90 \%$ of the 42 patients in the standard-therapy group compared with $28 \%$ of the 39 patients in the shortcourse therapy group. In addition, for patients whose CPIS remained $\leqslant 6$ on day 3 , antibiotics were nevertheless continued for $96 \%$ of the standard-therapy patients as opposed to $0 \%$ of the patients in the short-course group. Such a significant difference in antibiotic use between the two groups confirms that the experimental strategy was actually different from standard therapy, even though the diagnostic technique used was the same. Mortality and length of ICU stay did not differ between the two groups. Antimicrobial resistance, super infections, or both, developed in significantly lower percentages in the short-course antibiotic therapy group. Despite several limitations (the choice of ciprofloxacin, the studied population had a low likelihood of infection, the unknown 
modalities of standard treatment), the results of that study suggest that, based on a clinical strategy alone, overtreatment is highly prevalent, but unnecessary in most patients. Thus, it is possible to use a strategy leading to significantly lower antibiotic consumption without affecting the outcome of ventilated patients with clinical signs of pneumonia.

\section{Clinical strategy using qualitative cultures of tracheal secretions}

This approach is probably the most commonly applied in the ICU. In fact, it is used to complement a strategy based on clinical evaluation, with the objective to aid in the choice of antimicrobial agent(s). Curiously, this strategy has been poorly investigated and the studies conducted to evaluate qualitative cultures of tracheal secretions have provided disappointing results. In patients with histologically documented pneumonia, endotracheal aspirate sensitivity was $82 \%$ but its specificity was only $27 \%$, leading to overdiagnosis and overtreatment [24]. These cultures contribute to the diagnosis of VAP only when they are completely negative for a patient who has had new antibiotics introduced or no modification of ongoing antimicrobial treatment [25].

\section{Clinical strategy with quantitative cultures of tracheal secretions}

While simple qualitative cultures of endotracheal aspirates have a high percentage of false-positive results, the data obtained in some studies that used quantitative culture techniques suggest that endotracheal aspirate cultures may have an acceptable overall diagnostic accuracy [26-30], with a sensitivity of $82 \%$ and a specificity of $83 \%$ in one study, that established $10^{6}$ colony forming units $(\mathrm{cfu}) \cdot \mathrm{mL}^{-1}$ as the cutoff point [30]. However, when this threshold was applied, almost one-third of the patients with pneumonia were not identified and only $40 \%$ of the microorganisms cultured in endotracheal aspirate samples coincided with those obtained from PSB specimens [28]. Many authors have emphasised that microbiological results cannot be used to infer which microorganisms present in the trachea are really present in the lungs. In a study comparing quantitative endotracheal aspirate culture results with post mortem quantitative lung biopsy cultures, only $53 \%$ of microorganisms isolated from the trachea at concentrations $>10^{7} \mathrm{cfu} \cdot \mathrm{mL}^{-1}$ were also found in lung tissue [31].

Quantitative cultures of endotracheal aspirates may be an acceptable tool for diagnosing pneumonia when fibreoptic bronchoscopy techniques are not available. However, the major limitation of possible unnecessary antibiotic therapy or overtreatment with broad-spectrum antimicrobial agent persists.

\section{Clinical or bacteriological strategy using nonbronchoscopic techniques}

At least 15 studies have described a variety of nonbronchoscopic techniques for sampling lower respiratory tract secretions [32-37]. Potential advantages of these techniques are: less invasiveness with fewer gas-exchange perturbations because the endotracheal tube, which bypasses the proximal airways, permits easy and rapid access to the lower airways; its availability to nonbronchoscopists; its lower initial cost than bronchoscopy; and its applicability to patients intubated with small tubes. Acceptable results were obtained with these techniques by several investigators. Protected telescoping catheter, mini-BAL or protected mini-BAL, performed blindly, gave results similar to those obtained with the PSB techniques $[38,39]$. However, the overall concordance between blinded and bronchoscopic techniques was only $\sim 80 \%$, suggesting that the diagnosis could be missed, particularly in the case of pneumonia involving the left lung and/or the upper lobes $[35,40]$.

Finally, these techniques have not been evaluated as the bacteriological reference to manage patients suspected of having VAP.

\section{Bacteriological strategy using fibreoptic bronchoscopy to obtain protected specimen brush andlor bronchoalveolar lavage samples}

The use of a strategy based on bacteriological techniques such as quantitative cultures of PSB or BAL samples, helps guide the choice of antibiotic therapy while culture results precisely identify the offending organism(s) with good sensitivity, and avoids treatment of patients without pneumonia. Such data are invaluable for optimal antibiotic selection; they increase the intensivists confidence in managing patients with suspected nosocomial pneumonia [41]. Because of their potentially higher specificity than a clinical strategy, bronchoscopic techniques should reduce antibiotic-selection pressure in the ICU [11-13, 42], thereby limiting the emergence of drug-resistant strains, and the corresponding higher risks of superinfections [12, 42], thereby reducing overall costs, minimising antibiotic-related toxicity and, by directing attention away from the lung in the case of negative results, the search for a nonpulmonary infection is facilitated by the absence of empirical antimicrobial therapy that can mask the true diagnosis [43].

The potential contribution of the so-called invasive diagnostic techniques, more aptly referred to as bacteriological strategy to evaluate ventilated patients suspected of having developed VAP has been extensively investigated in both human and animal studies. Results of those analyses confirmed the following points: 1) PSB and BAL offer sensitive and specific approaches to differentiate between colonisation and distal lung infection, and to identify the microorganisms involved in pneumonia. Pooling the results of 18 studies evaluating the PSB technique showed a sensitivity of $89 \%$ and a specificity of $94 \%[14,44]$. In another study, when the results of 23 studies evaluating BAL were pooled, similar conclusions were drawn, with a sensitivity of $73 \%$ and a specificity of $82 \%$ [45]. 2) Bronchoscopic PSB and BAL are well-tolerated by severely ill ventilated patients, including patients with severe hypoxaemia, acute respiratory distress syndrome thrombocytopenia, severe head injury, etc. [33, 46-49]. 3) No increased mortality was observed in patients with suspected VAP who had antibiotics withdrawn on the basis of negative results of bronchoscopic techniques [50, 51]. 4) A bacteriological strategy is justified only if bronchoscopic techniques are performed in the absence of recent introduction or modification of antimicrobial therapy. The results of respiratory secretion cultures are usually not modified when pneumonia develops as a superinfection in patients who have been receiving systemic antibiotics for several days, because the bacteria responsible for the new infection have become resistant to the antibiotics being given [52, 53].

Five studies evaluated the impact of a diagnostic strategy using bronchoscopic specimens on the outcome of patients suspected of having VAP [41, 54-57]. Three Spanish randomised trials failed to demonstrate differences in mortality and morbidity [54, 55, 57]. However those studies, which 
compared PSB and/or BAL to quantitative or qualitative cultures of endotracheal aspirates, also applied the same clinical strategy to both arms: treatment of all suspected-VAP patients was not based on the results of bacteriological samples and antibiotics were continued in all patients despite negative results. As a consequence, in the study by SoLEVIOLAN et al. [57], antibiotic consumption was the same for both patients (mean duration of antimicrobial therapy was $13 \pm 4$ days for patients managed with PSB or BAL and $14 \pm 4$ days for those managed with endotracheal aspirates). No data on duration of antibiotic treatment were available in the two other studies [54, 55]. In addition, those studies were based on relatively small populations $(\mathrm{n}=51,76$ and 88$)$.

One of the first studies to clearly demonstrate a benefit in favour of the bacteriological strategy was a prospective cohort study conducted in 10 Canadian ICUs [58]. The authors compared 92 patients suspected of having developed pneumonia who underwent fibreoptic bronchoscopy and 49 patients who did not. Mortality among bronchoscopy patients was $19 \%$ versus $35 \%$ for controls $(\mathrm{p}=0.03)$. Furthermore, patients managed with a bacteriological strategy received fewer antibiotics and more patients had all their antibiotics discontinued compared to the clinical strategy group, thereby confirming that the two strategies actually differed.

A large, prospective, randomised trial compared clinical versus bacteriological strategy for the management of 413 patients suspected of having VAP [56]. The clinical strategy included empirical antimicrobial therapy, based on clinical evaluation and the presence of bacteria on direct examination of tracheal aspirates, and possible subsequent adjustment or discontinuation according to the results of qualitative cultures of endotracheal aspirates. The bacteriological strategy consisted of fibreoptic bronchoscopy with direct examination of BAL and/or PSB samples and empirical therapy initiated only when results were positive; a definitive diagnosis based on quantitative culture results of samples obtained with PSB or BAL was awaited before adjusting, discontinuing, or, for some patients with negative direct examination (no bacteria identified on cytocentrifuge preparation of BAL fluid, or PSB samples) and positive quantitative cultures $\left(>10^{3} \mathrm{cfu} \cdot \mathrm{mL}^{-1}\right.$ for the PSB and $>10^{4} \mathrm{cfu} \cdot \mathrm{mL}^{-1}$ for BAL), starting therapy. Empirical antimicrobial therapy was initiated in $91 \%$ of the patients in the clinical strategy group and in only $52 \%$ of those in the bacteriological strategy group. Compared with patients managed clinically, those receiving bacteriological management had a lower mortality rate on day $14(25 \%$ and $16 \%$; $\mathrm{p}=0.02$ ), lower sepsis-related organ failure assessment scores on day 3 and $7(\mathrm{p}=0.04)$, and less antibiotic use (mean number of antibiotic-free days, $2 \pm 3$ and $5 \pm 5 ; \mathrm{p}<0.001$ ). Multivariate analysis showed a significant difference in mortality on day 28 in favour of bacteriological management, associated with a significant reduction of antibiotic consumption. Pertinently, 22 nonpulmonary infections were diagnosed in the bacteriological strategy group and only five in the clinical strategy group, suggesting that overestimation of VAP may lead to missed nonpulmonary infections. The possible consequences of delayed treatment or definite diagnosis due to antibiotic interference are prolonged antibiotic therapy, more antibioticassociated complications, and induction of additional organ dysfunctions.

Thus, the implementation of bacteriological management for the diagnosis of VAP may lower antibiotic consumption and improve patient outcome. In clinical practice, it is most important that the physicians using these techniques establish a protocol supported by the literature and within the capabilities of the local microbiology laboratory. The choice of PSB or BAL may eventually depend on the preferences and experiences of individual physicians and the patient's underlying disease. Most investigators prefer BAL over PSB (slightly higher sensitivity and less costly test, most accurate direct examination with the use of cytocentrifuge preparations, possibility to diagnose nonbacterial pulmonary infection, etc.). The potential benefit of a microbiological strategy can be obtained only when physicians accept to prescribe antibiotics based on the results of bronchoscopic specimen cultures and, thus, to withdraw antimicrobial therapy from patients with negative results [58, 59].

When fibreoptic bronchoscopy is not available or contraindicated, or in the case of septic shock requiring immediate antibiotic administration, fibreoptic bronchoscopy can be replaced by a nonbronchoscopic diagnostic procedure, or the bacteriological strategy can be replaced by the CPIS strategy described by SINGH et al. [23] with re-evaluation 3 days later.

\section{Antimicrobial Treatment}

The choice and modalities of antibiotic treatment of VAP are complex issues. No consensus has been reached concerning issues as basic as the optimal antibiotic regimen or its duration.

Some general principles can be helpful for the selection of initial treatment: 1) knowledge of pathogens most frequently identified as responsible for VAP and their susceptibility patterns as determined by continuous collection of epidemiological surveillance data; 2) prior duration of hospitalisation and mechanical ventilation before the onset of VAP (early versus late VAP); 3) antibacterial activities of previously prescribed antibiotics; 4) information obtained by direct examination of pulmonary secretions; 5) intrinsic antibacterial activity and pharmacodynamic characteristics of each agent that could be used to treat this infection.

Although tracheal colonisation by potentially pathogenic microorganisms precedes lung infection in a majority of, but not all, ventilated patients, recent data have also emphasised that the pattern of tracheobronchial colonisation, and especially the types of microorganisms involved, reflect a dynamic process, with rapid modification of the flora present at that level and is influenced by factors such as prior duration of MV and prior antibiotics. In one study in which lower respiratory tract colonisation and infection were prospectively evaluated in 30 patients with severe ARDS, using repeated quantitative cultures of plugged telescopic catheter specimens taken blindly via the endotracheal tube every 48 to $72 \mathrm{~h}$ after ARDS onset, colonisation preceded BAL, VAP was only microbiologically confirmed in $67 \%$ of the VAP episodes [60]. Therefore, careful evaluation of distal airways colonisation can fail to document at least one-third of VAP episodes. Such a strategy may also considerably increase the workload of the microbiology laboratory without having any positive impact on patient management.

Colonisation with potentially drug-resistant pathogens, such as methicillin-resistant Staphlococcus aureas (MRSA) or extended-spectrum $\beta$-lactamase-producing strains of Klebsiella pneumoniae or other Enterobacteriaceae, is associated with an increased risk of infection caused by the corresponding microorganism. These results were confirmed in the study by HAYON et al. [61], with positive-predictive values of recovering such a microorganism from a specimen of $62 \%, 52 \%$ or $24 \%$ for VAP caused by MRSA, Pseudomonas aeruginosa or Acinetobacer baumannii, respectively. However, because the sensitivity of prior microbiological culture results for identifying bacteria causing VAP do not exceed $70 \%$, selection of initial antimicrobial therapy for patients with VAP can hardly be based only on these results, especially for deciding to use (or not) vancomycin and/or a broad-spectrum 
$\beta$-lactam effective against $P$. aeruginosa and/or A. baumannii. However, when one of the three microorganisms (or any pathogen) is isolated from respiratory secretions within $72 \mathrm{~h}$ of VAP, it should probably be covered by the antimicrobial regimen selected, even though predictive values do not exceed $50-60 \%$.

Taking these epidemiological characteristics into account may allow a rational decision-tree to be devised for selecting initial treatment in this setting that prevents resorting to broad-spectrum drug coverage in all patients. For example, monotherapy with a second-generation cephalosporin (cefuroxime, cefamandole, cefotetan), or a third-generation cephalosporin with no antipseudomonal activity (cefotaxime, ceftriaxone), or therapy combining the $\beta$-lactamase inhibitor, clavulanic acid, with amoxicillin would generally be an appropriate choice for most patients with early-onset VAP who have not received prior antimicrobial treatment. In contrast, for patients who have required prolonged MV and antimicrobial treatment, three-antibiotic therapy with a combination of aminoglycoside or ciprofloxacin plus a very broad-spectrum antipseudomonas $\beta$-lactam, such as piperacillin-tazobactam or imipenem, plus vancomycin should be started, keeping in mind that even such a regimen will not assure complete coverage of all putative pathogens. For the two intermediate groups, early-onset episodes with previous antibiotic therapy and late-onset episodes without previous antibiotic therapy, in which a mixed distribution of pathogens is frequently observed, including some nonfermenting Gramnegative bacilli, such as $P$. aeruginosa, but practically no MRSA and no multiresistant $A$. baumannii, treatment should be based on a combination of aminoglycoside or ciprofloxacin and an antipseudomonas $\beta$-lactam, but without vancomycin [61].

However, because the range of bacteria that cause VAP and their susceptibility patterns vary widely among hospitals in the same or different countries, selection of initial antimicrobial therapy needs to be tailored to each institution's local patterns of antimicrobial resistance. A computerised decisionsupport program linked to computer-based patient records can facilitate the dissemination of such information to physicians for immediate use in therapy decision-making and improve the quality of care. Of course, as soon as bacteriological results are known, antibiotic de-escalation should be performed and broad-spectrum agents be used only when required by the susceptibility patterns of the responsible pathogens.

Effective antibiotic treatment of bacterial pneumonia depends on adequate delivery of antibacterial agents to the infection site, and, therefore, scrupulous attention must be given to optimal doses, routes of administration and pharmacodynamic characteristics of each agent used to treat this infection. Antibiotic levels in infected tissues are considered to be therapeutic when free-drug concentrations equal at least the in vitro minimal inhibitory concentration (MIC) for the infecting pathogen(s). Due to major methodological problems, published data concerning the penetration of most antibiotics into the lung should probably be viewed with caution, and only general trends concerning concentrations achievable at the infected site in lung tissue can be derived from these studies.

Several published reports have demonstrated a relationship among serum concentrations of $\beta$-lactams or other antibiotics, the MIC of the infecting organism and the rate of bacterial eradication from respiratory secretions in patients with lung infection, thereby emphasising that clinical and bacteriological outcomes can be improved by optimising the therapeutic regimen according to pharmacokinetic properties of the agent(s) selected for treatment. Most investigators distinguish between antimicrobial agents that kill by a concentration-dependent mechanism (e.g. aminoglycosides and fluoroquinolones) from those that kill by a timedependent mechanism (e.g. $\beta$-lactams and vancomycin). Multivariate analyses based on 74 acutely ill, mostly VAP patients, who were treated with intravenous ciprofloxacin (200 mg b.i.d. to $400 \mathrm{mg}$ t.i.d.), demonstrated that the most important independent factor for probability of cure was a pharmacodynamic variable, i.e. the 24-h area under the concentration/time curve divided by the MIC (AUIC) [62]. For AUIC $<125$, the probabilities of clinical and microbiological cures were 42 and $26 \%$, respectively, but with AUIC $>125$, the probabilities were 80 and $82 \%$, respectively.

Pharmacokinetic/pharmacodynamic models have also been used to optimise aminoglycoside therapy for VAP caused by Gram-negative bacilli [63]. Seventy-eight patients with VAP were analysed, and the investigators reported an $89 \%$ success rate for temperature normalisation by day 7 of therapy for maximum concentration of drug in serum $(\mathrm{Cmax}) / \mathrm{MIC}>4.7$, and an $86 \%$ success rate for leukocyte count normalisation by day 7 of therapy for $\mathrm{Cmax} / \mathrm{MIC}>4.5$. Logistic regression analysis predicted a $90 \%$ probability of temperature and leukocyte count normalisations by day 7 , if a $\mathrm{Cmax} / \mathrm{MIC}>10$ was achieved within the first $48 \mathrm{~h}$ of aminoglycoside administration. Aggressive aminoglycoside doses immediately followed by pharmacokinetic monitoring for each patient would ensure that $\mathrm{Cmax} / \mathrm{MIC}$ target ratios are achieved early during therapy.

These findings confirm the need for adjusting the target dose of antimicrobial agents used to treat severe pulmonary infection to an individual patient's pharmacokinetics and putative bacterial pathogens' susceptibilities. Development of a priori dosing algorithms based on MIC, patient creatinine clearance and weight, and the clinician-specified AUIC target might therefore be a valid way to improve treatment of these patients, leading to a more precise approach than current guidelines for optimal use of antimicrobial agents.

Further trials are needed to clarify uncertainties concerning the alternative between monotherapy and combination therapy, the optimal duration of treatment, and the exact place of antibiotic rotation. In the meantime, it is probably safer to use a $\beta$-lactam antibiotic in combination with an aminoglycoside or a quinolone for patients with severe VAP, at least for the first days of therapy, while culture results of pulmonary secretions are pending. It may be that monodrug therapies for nosocomial pneumonia would best be reserved for infections in which $P$. aeruginosa or other multiresistant microorganisms, such as Klebsiella, Enterobacter, Citrobacter, Serratia or Acinetobacter spp. have been excluded as the etiological agents.

To conclude, it should be emphasised that, for the management of ventilator-associated pneumonia, as for all infectious diseases, the choice of antimicrobial treatment is much easier when the specific etiological agent(s) is identified by a reliable diagnostic technique. Before new antibiotics are administered, reliable pulmonary specimens must be obtained, for direct examination and cultures, from patients clinically suspected of having developed ventilator-associated pneumonia.

\section{References}

1. Kollef MH, Sherman G, Ward S, Fraser VJ. Inadequate antimicrobial treatment of infections: a risk factor for hospital mortality among critically ill patients. Chest 1999; 115: 462-474.

2. Craven DE, Steger KA. Nosocomial pneumonia in mechanically ventilated adult patients: epidemiology and prevention in 1996. Semin Respir Infect 1996; 11: 32-53.

3. American Thoracic Society. Hospital-acquired pneumonia in adults: diagnosis, assessment of severity, initial antimicrobial 
therapy, and preventive strategies. A consensus statement, American Thoracic Society, November 1995. Am J Respir Crit Care Med 1996; 153: 1711-1725.

4. Johanson WG, Pierce AK, Sanford JP, Thomas GD. Nosocomial respiratory infections with gram-negative bacilli. The significance of colonization of the respiratory tract. Ann Intern Med 1972; 77: 701-706.

5. Garrouste-Orgeas M, Chevret S, Arlet G, et al. Oropharyngeal or gastric colonization and nosocomial pneumonia in adult intensive care unit patients. A prospective study based on genomic DNA analysis. Am J Respir Crit Care Med 1997; 156: $1647-1655$.

6. Bonten MJ, Gaillard CA, de Leeuw PW, Stobberingh EE. Role of colonization of the upper intestinal tract in the pathogenesis of ventilator-associated pneumonia. Clin Infect Dis 1997; 24: 309-319.

7. Andrews CP, Coalson JJ, Smith JD, Johanson WG. Diagnosis of nosocomial bacterial pneumonia in acute, diffuse lung injury. Chest 1981; 80: 254-258.

8. Meduri GU, Mauldin GL, Wunderink RG, et al. Causes of fever and pulmonary densities in patients with clinical manifestations of ventilator-associated pneumonia. Chest 1994; 106: 221-235.

9. Wunderink RG, Woldenberg LS, Zeiss J, Day CM, Ciemins $J$, Lacher DA. The radiologic diagnosis of autopsy-proven ventilator-associated pneumonia. Chest 1992; 101: 458-463.

10. Baselski V. Microbiologic diagnosis of ventilator-associated pneumonia. Infect Dis Clin North Am 1993; 7: 331-357.

11. Kollef MH. Ventilator-associated pneumonia. A multivariate analysis. JAMA 1993; 270: 1965-1970.

12. McGowan JE. Antimicrobial resistance in hospital organisms and its relation to antibiotic use. Rev Infect Dis 1983; 5: 1033-1048.

13. Neu HC. The crisis in antibiotic resistance. Science 1992; 257: 1064-1073.

14. Chastre J, Fagon JY. Ventilator-associated pneumonia. Am J Respir Crit Care Med 2002; 165: 867-903.

15. Waterer GW, Wunderink RG. Controversies in the diagnosis of ventilator-associated pneumonia. Med Clin North Am 2001; 85: 1565-1581.

16. Fabregas N, Ewig S, Torres A, et al. Clinical diagnosis of ventilator associated pneumonia revisited: comparative validation using immediate postmortem lung biopsies. Thorax 1999; 54: 867-873.

17. Trouillet JL, Chastre J, Vuagnat A, et al. Ventilatorassociated pneumonia caused by potentially drug-resistant bacteria. Am J Respir Crit Care Med 1998; 157: 531-539.

18. Hanberger $\mathrm{H}$, Garcia-Rodriguez JA, Gobernado $M$, Goossens H, Nilsson LE, Struelens MJ. Antibiotic susceptibility among aerobic Gram-negative bacilli in intensive care units in 5 European countries. French and Portuguese ICU Study Groups. JAMA 1999; 281: 67-71.

19. Fagon JY, Chastre J, Domart Y, et al. Nosocomial pneumonia in patients receiving continuous mechanical ventilation. Prospective analysis of 52 episodes with use of a protected specimen brush and quantitative culture techniques. Am Rev Respir Dis 1989; 139: 877-884.

20. Rello J, Sa-Borges M, Correa H, Leal SR, Baraibar J. Variations in etiology of ventilator-associated pneumonia across four treatment sites: implications for antimicrobial prescribing practices. Am J Respir Crit Care Med 1999; 160: 608-613.

21. Ibrahim EH, Ward S, Sherman G, Schaiff R, Fraser VJ, Kollef MH. Experience with a clinical guideline for the treatment of ventilator-associated pneumonia. Crit Care Med 2001; 29: 1109-1115.

22. Pugin J, Auckenthaler R, Mili N, Janssens JP, Lew PD, Suter PM. Diagnosis of ventilator-associated pneumonia by bacteriologic analysis of bronchoscopic and nonbronchoscopic "blind" bronchoalveolar lavage fluid. Am Rev Respir Dis 1991; 143: 1121-1129.
23. Singh N, Rogers $\mathrm{P}$, Atwood CW, Wagener MM, Yu VL Short-course empiric antibiotic therapy for patients with pulmonary infiltrates in the intensive care unit. A proposed solution for indiscriminate antibiotic prescription. Am J Respir Crit Care Med 2000; 162: 505-511.

24. Hill JD, Ratliff JL, Parrott JC, et al. Pulmonary pathology in acute respiratory insufficiency: lung biopsy as a diagnostic tool. J Thorac Cardiovasc Surg 1976; 71: 64-71.

25. Kirtland SH, Corley DE, Winterbauer RH, et al. The diagnosis of ventilator-associated pneumonia: a comparison of histologic, microbiologic, and clinical criteria. Chest 1997; 112: 445-457.

26. Marquette $\mathrm{CH}$, Copin $\mathrm{MC}$, Wallet $\mathrm{F}$, et al. Diagnostic tests for pneumonia in ventilated patients: prospective evaluation of diagnostic accuracy using histology as a diagnostic gold standard. Am J Respir Crit Care Med 1995; 151: 1878-1888.

27. Sauaia A, Moore FA, Moore EE, Haenel JB, Kaneer L, Read RA. Diagnosing pneumonia in mechanically ventilated trauma patients: endotracheal aspirate versus bronchoalveolar lavage. J Trauma 1993; 35: 512-517.

28. Jourdain B, Novara A, Joly-Guillou ML, et al. Role of quantitative cultures of endotracheal aspirates in the diagnosis of nosocomial pneumonia. Am J Respir Crit Care Med 1995; 152: 241-246.

29. Cook D, Mandell L. Endotracheal aspiration in the diagnosis of ventilator-associated pneumonia. Chest 2000; 117: Suppl. 1, 195S-197S.

30. Marquette $\mathrm{CH}$, Georges $\mathrm{H}$, Wallet $\mathrm{F}$, et al. Diagnostic efficiency of endotracheal aspirates with quantitative bacterial cultures in intubated patients with suspected pneumonia. Comparison with the protected specimen brush. Am Rev Respir Dis 1993; 148: 138-144.

31. Borderon E, Leprince A, Gueveler C, Borderon JC. The diagnostic value of quantitative bacteriology in tracheal aspirates compared to lung biopsy (in French). Rev Fr Mal Respir 1981; 9: 229-239.

32. Rouby JJ, Martin de Lassale E, Poete P, et al. Nosocomial bronchopneumonia in the critically ill. Histologic and bacteriologic aspects. Am Rev Respir Dis 1992; 146: 10591066.

33. Kollef MH, Bock KR, Richards RD, Hearns ML. The safety and diagnostic accuracy of minibronchoalveolar lavage in patients with suspected ventilator-associated pneumonia. Ann Intern Med 1995; 122: 743-748.

34. A'Court CH, Garrard CS, Crook D, et al. Microbiological lung surveillance in mechanically ventilated patients, using non-directed bronchial lavage and quantitative culture. $Q J$ Med 1993; 86: 635-648.

35. Jorda R, Parras F, Ibanez J, Reina J, Bergada J, Raurich JM. Diagnosis of nosocomial pneumonia in mechanically ventilated patients by the blind protected telescoping catheter. Intensive Care Med 1993; 19: 377-382.

36. Papazian L, Thomas P, Garbe L, et al. Bronchoscopic or blind sampling techniques for the diagnosis of ventilatorassociated pneumonia. Am J Respir Crit Care Med 1995; 152: 1982-1991.

37. Leal-Noval SR, Alfaro-Rodriguez E, Murillo-Cabeza F, Garnacho-Montero J, Rey-Perez J, Munoz-Sanchez MA. Diagnostic value of the blind brush in mechanically ventilated patients with nosocomial pneumonia. Intensive Care Med 1992; 18: 410-414.

38. Pham LH, Brun-Buisson C, Legrand P, et al. Diagnosis of nosocomial pneumonia in mechanically ventilated patients. Comparison of a plugged telescoping catheter with the protected specimen brush. Am Rev Respir Dis 1991; 143: $1055-1061$.

39. Bregeon F, Papazian L, Thomas P, et al. Diagnostic accuracy of protected catheter sampling in ventilatorassociated bacterial pneumonia. Eur Respir $J$ 2000; 16: 969-975.

40. Meduri GU, Reddy RC, Stanley T, El-Zeky F. Pneumonia in acute respiratory distress syndrome. A prospective 
evaluation of bilateral bronchoscopic sampling. Am J Respir Crit Care Med 1998; 158: 870-875.

41. Heyland DK, Cook DJ, Marshall J, et al. The clinical utility of invasive diagnostic techniques in the setting of ventilatorassociated pneumonia. Canadian Critical Care Trials Group. Chest 1999; 115: 1076-1084.

42. Kollef MH, Fraser VJ. Antibiotic resistance in the intensive care unit. Ann Intern Med 2001; 134: 298-314.

43. Liu YC, Huang WK, Huang TS, Kunin CM. Inappropriate use of antibiotics and the risk for delayed admission and masked diagnosis of infectious diseases: a lesson from Taiwan. Arch Intern Med 2001; 161: 2366-2370.

44. Torres A, Fabregas N, Ewig S, de la Bellacasa JP, Bauer TT, Ramirez J. Sampling methods for ventilator-associated pneumonia: validation using different histologic and microbiological references. Crit Care Med 2000; 28: 2799-2804.

45. Torres A, El-Ebiary M. Bronchoscopic BAL in the diagnosis of ventilator-associated pneumonia. Chest 2000; 117: Suppl. 2, 198S-202S.

46. Montravers P, Gauzit R, Dombret MC, Blanchet F, Desmonts JM. Cardiopulmonary effects of bronchoalveolar lavage in critically ill patients. Chest 1993; 104: 1541-1547.

47. Trouillet JL, Guiguet M, Gibert C, et al. Fiberoptic bronchoscopy in ventilated patients. Evaluation of cardiopulmonary risk under midazolam sedation. Chest 1990; 97 : 927-933.

48. Steinberg KP, Mitchell DR, Maunder RJ, Milberg JA, Whitcomb ME, Hudson LD. Safety of bronchoalveolar lavage in patients with adult respiratory distress syndrome. Am Rev Respir Dis 1993; 148: 556-561.

49. Meduri GU, Belenchia JM, Estes RJ, Wunderink RG, el Torky M, Leeper KV KV Jr. Fibroproliferative phase of ARDS. Clinical findings and effects of corticosteroids. Chest 1991; 100: 943-952.

50. Croce MA, Fabian TC, Waddle-Smith L, et al. Utility of Gram's stain and efficacy of quantitative cultures for posttraumatic pneumonia: a prospective study. Ann Surg 1998; 227: 743-751.

51. Bonten MJ, Bergmans DC, Stobberingh EE, et al. Implementation of bronchoscopic techniques in the diagnosis of ventilator-associated pneumonia to reduce antibiotic use. Am J Respir Crit Care Med 1997; 156: 1820-1824.

52. Montravers P, Fagon JY, Chastre J, et al. Follow-up protected specimen brushes to assess treatment in nosocomial pneumonia. Am Rev Respir Dis 1993; 147: 38-44.
53. Johanson WG Jr, Seidenfeld JJ, Gomez P, de los Santos R, Coalson JJ. Bacteriologic diagnosis of nosocomial pneumonia following prolonged mechanical ventilation. Am Rev Respir Dis 1988; 137: 259-264.

54. Sanchez-Nieto JM, Torres A, Garcia-Cordoba F, et al. Impact of invasive and noninvasive quantitative culture sampling on outcome of ventilator-associated pneumonia: a pilot study. Am J Respir Crit Care Med 1998; 157: 371-376.

55. Ruiz M, Torres A, Ewig S, et al. Noninvasive versus invasive microbial investigation in ventilator-associated pneumonia: evaluation of outcome. Am J Respir Crit Care Med 2000; 162: 119-125.

56. Fagon JY, Chastre J, Wolff $\mathrm{M}$, et al. Invasive and noninvasive strategies for management of suspected ventilator-associated pneumonia. A randomized trial. Ann Intern Med 2000; 132: 621-630.

57. Sole-Violan J, Fernandez JA, Benitez AB, Cardenosa Cendrero JA, Rodriguez de Castro F. Impact of quantitative invasive diagnostic techniques in the management and outcome of mechanically ventilated patients with suspected pneumonia. Crit Care Med 2000; 28: 2737-2741.

58. Baker AM, Bowton DL, Haponik EF. Decision making in nosocomial pneumonia. An analytic approach to the interpretation of quantitative bronchoscopic cultures. Chest 1995; 107: 85-95.

59. Sterling TR, Ho EJ, Brehm WT, Kirkpatrick MB. Diagnosis and treatment of ventilator-associated pneumonia - impact on survival. A decision analysis. Chest 1996; 110: 1025-1030.

60. Delclaux C, Roupie E, Blot F, Brochard L, Lemaire F, BrunBuisson C. Lower respiratory tract colonization and infection during severe acute respiratory distress syndrome: incidence and diagnosis. Am J Respir Crit Care Med 1997; 156: 1092-1098.

61. Hayon J, Figliolini C, Combes A, et al. Role of serial routine microbiologic culture results in the initial management of ventilator-associated pneumonia. Am J Respir Crit Care Med 2002; 165: 41-46.

62. Forrest A, Nix DE, Ballow CH, Goss TF, Birmingham MC, Schentag JJ. Pharmacodynamics of intravenous ciprofloxacin in seriously ill patients. Antimicrob Agents Chemother 1993; 37: 1073-1081.

63. Kashuba AD, Nafziger AN, Drusano GL, Bertino JS Jr. Optimizing aminoglycoside therapy for nosocomial pneumonia caused by gram-negative bacteria. Antimicrob Agents Chemother 1999; 43: 623-629. 